\section{Infarto agudo de miocardio sin enfermedad coronaria ateroesclerótica obstructiva: utilidad de las imágenes intravasculares y resonancia cardíaca en su diagnóstico}

\author{
PABLA CATALDO V. ${ }^{1,2, a}$, FERNANDO J. VERDUGO ${ }^{1, a}$, \\ CHRISTIAN DAUVERGNE ${ }^{1,3}$, ALFONSO GARCÍA ${ }^{1, b}$, PABLO ANTILEO $^{1, b}$, \\ RODRIGO MONSALVE ${ }^{1, \mathrm{~b}}$, FERNANDO PINEDA ${ }^{1}$, MANUEL MÉNDEZ ${ }^{1}$, \\ POLENTZI URIARTE ${ }^{1}$, MARIO ARAYA H. ${ }^{3}$, PEDRO LLERENA ${ }^{3, c}$, \\ YALILE NAUHM ${ }^{3, c}$, GONZALO PEREIRA ${ }^{2}$, CRISTÓBAL RAMOS ${ }^{1,3}$, \\ MARCO COELLO V. ${ }^{2}$, CHRISTIAN GARRIDO G. ${ }^{1, \mathrm{~d}}$, \\ TOMAS DELGADO A. ${ }^{1, \mathrm{~d}}$, SOLEDAD GONZÁLEZ ${ }^{1, \mathrm{e}}$, JORGE SANDOVAL B. ${ }^{1}$
}

\section{Myocardial infarction with non-obstructive coronary artery disease. Diagnostic value of intravascular imaging and cardiac resonance}

Background: Myocardial infarction with non-obstructive coronary artery disease (MINOCA) is common. Cardiac magnetic resonance (CMR) and intravascular imaging (IVI) may be useful for establishing its etiology. Aim: To describe a population with MINOCA and its multi-image assessment using IVI or CMR. Material and Methods: Review of medical records, imaging and functional studies of patients with MINOCA treated in three different clinical centers between 2015 and 2019. Results: Twenty-eight patients with MINOCA and IVI were included. Seventy eight percent were women, $46 \%$ had hypertension, 32\% smoked and 32\% had dyslipidemia. At wall motion assessment, 46\% presented apical ballooning pattern. In 36\% of patients IVI identified lesions that explained the cause of MINOCA, namely plaque disruption (PD) in 18\%, spontaneous coronary dissection in $11 \%$ and a thrombus without PD in $7 \%$. Forty-six percent of patients had uncomplicated atherosclerotic plaques, and $36 \%$ had no pathological findings. CMR was performed in 50\% of patients, identifying in all a diagnostic pattern. In nine cases it was compatible with stress cardiomyopathy, three cases had a myocarditis and two cases had transmural infarctions. PD and transmural late gadolinium enhancement were observed in $23 \%$ of patients with apical ballooning. Patients with a pattern of myocarditis did not have acute pathological findings at IVI. After a mean follow-up of 16.4 \pm 11.4 months, 3 patients with PD died. Conclusions: Among patients with MINOCA, there was a predominance of female gender with low cardiovascular risk factor load. The multi-image assessment allowed greater precision for etiological diagnosis of MINOCA. Apical ballooning was not pathognomonic for stress cardiomyopathy. $P D$ was associated with mortality.

(Rev Med Chile 2020; 148: 1083-1089)

Key words: Coronary Angiography; Magnetic Resonance Imaging; Myocardial Infarction; Tomography, Optical Coherence; Ultrasonography, Interventional.

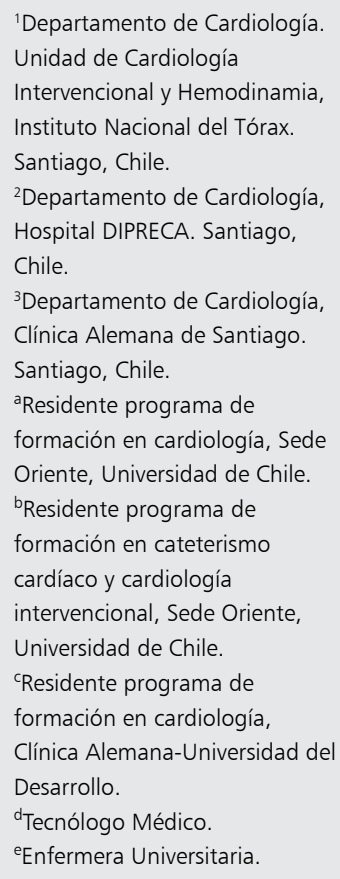

Trabajo no recibió financiamiento. Los autores declaran no tener conflictos de interés.

Recibido el 2 de febrero de 2020, aceptado el 15 de julio de 2020.

Correspondencia a: Dra. Pabla Cataldo Villarroel Instituto Nacional del Tórax, José Manuel Infante 717. Providencia. Santiago, Chile. pablacataldov@gmail.com 
$\mathrm{E}$ 1 infarto agudo de miocardio (IAM) es una de las principales causas de mortalidad; representando el $7,5 \%$ de las defunciones en Chile durante el 2018 ${ }^{1}$. Siguiendo las recomendaciones de guías clínicas actuales, los pacientes con IAM deben realizarse una coronariografía diagnóstica en forma precoz, con el objetivo de identificar y tratar oclusiones coronarias culpables $^{2,3}$. El IAM con ausencia de estenosis coronarias mayores de $50 \%$, denominado con el acrónimo de MINOCA ${ }^{4}$, es frecuente en la práctica clínica actual. Series internacionales han reportado una prevalencia entre 5 y $25 \%$ de las coronariografías diagnosticas realizadas por IAM ${ }^{5}$.

Clínicamente, la población de pacientes con MINOCA destaca por una mayor prevalencia en pacientes con el sexo femenino, menor edad al momento de presentación y una baja prevalencia factores de riesgo cardiovascular ${ }^{5-7}$, en comparación con pacientes con IAM con enfermedad coronaria obstructiva.

El MINOCA es un diagnóstico operativo, indica un mecanismo de daño miocárdico por múltiples posibles etiologías 8 incluyendo: disrupción de placa ateroesclerótica (DP), vasoespasmo coronario, disección coronaria espontánea, miocardiopatía por estrés (Síndrome de Takotsubo), miocarditis o embolia coronaria. Estas etiologías pueden ser de difícil diagnóstico y evidencia las limitaciones de la angiografía. Para identificar estas causas es necesario la utilización de imágenes intravasculares (IVI) y la resonancia magnética cardiaca (RMC). Las IVI, incluyendo ultrasonido intracoronario (IVUS) y tomografía de coherencia óptica (OCT), son técnicas complementarias a la coronariografía que se realizan durante el mismo procedimiento diagnóstico. Son imágenes de alta resolución axial (entre 20 a $100 \mu \mathrm{m}$ ), lo que permite identificar placas ateroscleróticas estables, accidentes de placas (ulceración, erosión, nódulos de calcio), trombos intracoronarios (blancos y rojos), disección coronaria espontánea o trombosis de dispositivos intravasculares. La RMC ha emergido como el método no invasivo de elección para la adecuada caracterización tisular del miocardio. Permite distinguir injuria miocárdica isquémica de no isquémica, determinar la presencia de edema, fibrosis o cicatriz, e inclusive cuantificar áreas de edema e inflamación mediante mapas paramétricos ${ }^{9}$.

El impacto de MINOCA sobre el pronóstico de los pacientes es controversial por las diversas etiologías, existiendo reportes de menor, igual o mayores tasas de mortalidad e infarto recurrente al compararse con pacientes con IAM con estenosis coronarias ateroscleróticas obstructivas $^{7,10-12}$.

El objetivo del presente estudio es caracterizar una población con MINOCA y su evaluación imagenológica multimodal mediante IVI y RMC, describiendo su evolución en mediano a largo plazo.

\section{Metodología}

Estudio descriptivo retrospectivo de pacientes atendidos en 3 centros asistenciales entre enero de 2015 y noviembre de 2019. Los criterios de inclusión fueron: 1) IAM con ausencia de estenosis coronarias significativas en coronariografía diagnóstica; 2) Realización de OCT o IVUS; 3) Evaluación de fracción de eyección del ventrículo izquierdo (FEVI) y motilidad segmentaria mediante ventriculografía y/o ecocardiograma transtorácico (ETT). Se excluyeron pacientes con intervenciones coronarias percutáneas o revascularización quirúrgica previa e IAM tipo $2^{8}$.

Se accedió a la ficha clínica y registraron datos clínicos. Se revisaron imágenes de coronariografía, IVI, ETT y RMC. La FEVI fue medida en ETT por Simpson biplano para el evento índice de MINOCA y a mediano plazo (4 a 9 meses post MINOCA). Acorde a los trastornos de motilidad segmentaria presentes en la ventriculografía y/o ETT, se clasificaron a los pacientes en:

1) Patrón epicardico: alteraciones de motilidad limitadas al territorio irrigado por una arteria coronaria epicárdica.

2) Patrón microvascular: alteraciones de motilidad extendidas más allá del territorio irrigado por una coronaria, o ausencia de trastornos de motilidad. Dentro de este, el patrón de balonamiento apical se definió por la presencia de hipocinesia/acinesia de segmentos apicales con hipermotilidad de segmentos basales.

Se realizó seguimiento telefónico de eventos cardiovasculares mayores, incluyendo: muerte, IAM, hospitalizaciones por causa cardiovascular y accidente cerebrovascular. Se cotejó con la ficha clínica y la base de datos del Registro Civil con fecha 31 de diciembre de 2019. Los eventos clínicos mayores fueron definidos según recomendaciones 
de la Standardized Data Collection for Cardiovascular Trials Initiative (SCTI) y la cuarta definición universal del Infarto de Miocardio ${ }^{8,13}$.

El estudio fue aprobado por el Comité de Ética Científico de Adultos del Servicio de Salud Metropolitano Oriente.

\section{Imágenes intravasculares}

Según el criterio de cada operador, se realizó OCT (Ilumien Terumo ${ }^{\circledR}$, Optis Abbot ${ }^{\circledR}$ ) con protocolo de adquisición de $20 \mathrm{~mm} /$ segundo, pullback de $\geq 60 \mathrm{~mm}$ de longitud; o IVUS (Volcano Revolution $45 \mathrm{mHz}^{\circledR}$; Ascist HDi, Kodama 60 $\mathrm{mHz}^{\circledR}$ ) con pullback $0,5 \mathrm{~mm} / \mathrm{seg}$, longitud de $\geq 60$ $\mathrm{mm}$. Las imágenes fueron evaluadas por operadores entrenados. Se consideró los hallazgos en IVI según recomendaciones de la European Association of Percutaneous Cardiovascular Interventions ${ }^{14}$ :

1) Disrupción de placa aterosclerótica (DP): Rotura de placa, nódulos calcificados o erosiones;

a) Rotura de placa: Solución de continuidad de la placa fibrosa con formación de una cavidad dentro de la placa.

b) Nódulo calcificado: Solución de continuidad de placa fibrosa de una placa calcificada con protrusión de calcificaciones o presencia de calcio proximal y/o distal a la lesión.

c) Erosión: Trombo en relación a placa aparente estable o intacta.

2) Trombo intracoronario: Masa irregular roja o blanca con diámetro $>250 \mu \mathrm{m}$ heterogénea intravascular.

3) Disección coronaria espontánea: Luz verdadera y luz falsa separadas por un flap intimo-medial con o sin puerta de entrada y ausencia de placa aterosclerótica. Se consideró como hallazgo patológico agudo a la presencia en IVI de cualquiera de las lesiones coronarias antes definidas.

\section{Resonancia magnética cardiaca}

Se realizaron secuencias de cine, T2 para evaluación de edema, y realce tardío con gadolinio en equipos de 1.5 Tesla. En base a estas secuencias, se clasificaron los pacientes en los siguientes patrones:

1) Patrón de Miocarditis (basado en cumplir $\geq 2 / 3$ criterios de Lake Louis): Edema en secuencia T2-STIR, realce precoz de gadolinio compatible con hiperemia y/o realce tardío con gadolinio parcheado centromiocárdico o subepicárdico ${ }^{15}$.
2) Patrón de Infarto: Presencia de realce tardío de gadolinio con compromiso transmural o subendocárdico en un territorio coronario ${ }^{16}$.

3) Patrón de miocardiopatía por estrés: Edema en secuencia T2-STIR, compromiso de la motilidad segmentaria característico que compromete más de un territorio coronario y ausencia de realce tardío con gadolinio ${ }^{16}$.

4) Normal: Ausencia de trastornos de motilidad segmentaria, edema o realce tardío de gadolinio ${ }^{16}$. La indicación de RMC en los pacientes fue determinada por el equipo tratante de cada centro asistencial, no existiendo una evaluación protocolizada.

\section{Análisis estadístico}

Las variables cuantitativas se expresan como media \pm desviación estándar (DE). Variables cualitativas se expresan en valor absoluto y porcentaje. Se realizó prueba t-student para variables cuantitativas y Chi-cuadrado para variables cualitativas. Los análisis se realizaron en IBM SPSS Statistics 20. Se consideró significativo un valor de $\mathrm{p}<0,05$.

\section{Resultados}

Se incluyó 28 pacientes con MINOCA. Las características basales del grupo total y subpoblaciones según patrón epicárdico o microvascular se describen en la Tabla 1. En nuestra serie la problación de sexo femenino correspondió al $78,6 \% ; 46,4 \%$ de hipertensión arterial; 32,1\% de dislipidemia; $32,1 \%$ de tabaquismo; y $25 \%$ de diabéticos. La presentación clínica fue angina típica en $89,3 \%$ y elevación del segmento ST en $46,4 \%$ de los pacientes.

En base a ventriculografía o ecocardiografía, se valoró la motilidad segmentaria en todos los pacientes, siendo catalogados en patrón microvascular 19 pacientes $(67,9 \%)$ y en patrón epicárdico 9 pacientes $(32,1 \%)$. Los pacientes con patrón epicárdico presentaron mayor edad y elevación del segmento ST $(p<0,05)$, sin exhibir diferencias en la prevalencia de comorbilidades. Se describió un trastorno de motilidad tipo balonamiento apical en 13 pacientes $(46,4 \%)$.

\section{Imágenes intravasculares}

Se realizó OCT en 25 pacientes (89,3\%) e IVUS en 3 pacientes (10,7\%). El IVI identificó en 35,7\% 
hallazgos patológicos agudos que explicaran la causa de MINOCA (17,85\% DP; 10,71\% disección coronaria espontánea; $7,14 \%$ trombo sin DP); 46,4\% presentaron placa aterosclerótica no complicada y $35,7 \%$ de los pacientes no tuvieron hallazgos patológicos en IVI.

Al considerar el uso de IVI en todos los pacientes, se logró establecer un diagnostico etiológico anatómico intravascular de MINOCA en 10 pacientes $(35,7 \%)$. El uso de IVI permitió un diagnostico etiológico en $77,8 \%$ de los pacientes con alteraciones de motilidad segmentaria de patrón epicardico. En pacientes con patrón microvascular, el uso de IVI permitió identificar hallazgos patológicos agudos sin requerimiento de otras imágenes en $22,2 \%$ de los pacientes.

\section{Resonancia magnética cardiaca}

Se realizó RMC en 14 pacientes con MINOCA, 12 pacientes con patrón microvascular $(63,1 \%)$ y 2 pacientes con patrón epicárdico $(22,2 \%)$. En los pacientes con patrón microvascular, $75 \%$ presentó patrón de miocardiopatía por estrés y $25 \%$ patrón de miocarditis. Los pacientes con patrón epicardico presentaron patrón de infarto transmural.

\section{Integración de hallazgos imagenológicos intravasculares y no invasivos}

La evaluación multimodal con IVI y RMC permitió un diagnostico imagenológico en 15 pacientes.

En los pacientes que presentaron patrón de balonamiento apical $(\mathrm{n}=13)$, la IVI identificó 5 pacientes con placas ateroscleróticas sin DP $(38,4 \%), 3$ pacientes con DP $(23,1 \%)$, y 5 pacientes sin placas ateroscleróticas $(38,4 \%)$.

Se corroboró la presencia de patrón de infarto transmural en todos los pacientes con DP que se realizaron RMC. Los pacientes sin DP no presentaron áreas de realce tardío compatibles con patrón de infarto en RMC.

En dos pacientes con trombos intracoronarios en ausencia de placa por IVI, la evaluación imagenológica no invasiva señaló que un paciente presentaba una miocardiopatía dilatada con trombo intracavitario, y en otro paciente evidencio un foramen oval permeable con probable embolia paradojal. Ambos hallazgos fueron sugerentes de embolia coronaria como etiología de MINOCA.

\section{Seguimiento}

La evolución de FEVI se exhibe en la Tabla 2. No hubo diferencias significativas de FEVI basal entre el grupo microvascular y epicardico. En el seguimiento, el grupo microvascular muestra un aumento promedio de $+9,2 \pm 10,8 \%(0-33 \%)$ ( $\mathrm{p}<0,01$ respecto a basal). No hubo diferencias significativas en el grupo epicárdico.

No hubo muertes intrahospitalarias durante el evento índice. Se realizó un seguimiento clínico, con media 16,4 $\pm 11,5$ meses (mediana 16,8). Los resultados de eventos cardiovasculares mayores, mortalidad, hospitalizaciones cardiovasculares e IAM recurrente se muestran en la Tabla 3. No

Tabla 1. Características basales de pacientes con MINOCA

\begin{tabular}{|lcccc|}
\hline Variable & $\begin{array}{c}\text { Total } \\
(\mathbf{n = 2 8 )}\end{array}$ & $\begin{array}{c}\text { Microvascular } \\
(\mathbf{n}=\mathbf{1 9})\end{array}$ & $\begin{array}{c}\text { Epicárdico } \\
(\mathbf{n}=\mathbf{9})\end{array}$ & p \\
\hline Edad promedio (años) & $61,3 \pm 16,9$ & $56,8 \pm 16,7$ & $70,9 \pm 13,8$ & 0,04 \\
Género (femenino) & $22(78,6 \%)$ & $16(84,2 \%)$ & $6(66,7 \%)$ & 0,29 \\
\hline Hipertensión arterial & $13(46,4 \%)$ & $11(57,9 \%)$ & $4(44,4 \%)$ & 0,50 \\
\hline Diabetes mellitus & $7(25,0 \%)$ & $4(21,1 \%)$ & $3(33,3 \%)$ & 0,48 \\
\hline Dislipidemia & $9(32,1 \%)$ & $5(26,3 \%)$ & $4(44,4 \%)$ & 0,34 \\
Tabaquismo & $9(32,1 \%)$ & $6(31,6 \%)$ & $3(33,3 \%)$ & 0,93 \\
Miocardiopatía por estrés previa & $3(10,7 \%)$ & $3(15,8 \%)$ & $0(0,0 \%)$ & 0,21 \\
\hline Angina típica & $25(92,8 \%)$ & $16(84,2 \%)$ & $9(100 \%)$ & 0,21 \\
\hline Elevación de segmento ST & $13(46,4 \%)$ & $6(31,6 \%)$ & $7(77,8 \%)$ & 0,02 \\
\hline BCRI & $2(7,1 \%)$ & $1(5,3 \%)$ & $1(11,1 \%)$ & 0,57 \\
\hline
\end{tabular}


Tabla 2. Función ventricular izquierda basal y al seguimiento medido por ETT

\begin{tabular}{|lcccc|}
\hline Patrón & FEVI basal & FEVI seguimiento & Delta & p \\
\hline Microvascular & $46,3 \pm 10,0(24-60)$ & $55,5 \pm 10,1(24-72)$ & $+9,2 \pm 10,8$ & 0,002 \\
\hline Epicardico & $45,2 \pm 13,3(30-67)$ & $49,2 \pm 15,2(25-66)$ & $+4,0 \pm 8,3$ & 0,186 \\
\hline
\end{tabular}

Tabla 3. Seguimiento eventos clínicos de pacientes con MINOCA

\begin{tabular}{|lcccc|}
\hline Variable & $\begin{array}{c}\text { Total } \\
(\mathbf{n = 2 8 )}\end{array}$ & $\begin{array}{c}\text { Microvascular } \\
(\mathbf{n}=\mathbf{1 9})\end{array}$ & $\begin{array}{c}\text { Epicárdico } \\
\text { (n = 9) }\end{array}$ & p \\
\hline Eventos cardiovasculares mayores & $8(28,5 \%)$ & $3(15,7 \%)$ & $5(55,5 \%)$ & 0,18 \\
Mortalidad & $3(10,7 \%)$ & $0(0,0 \%)$ & $3(33,3 \%)$ & 0,01 \\
Hospitalización cardiovascular & $7(25,0 \%)$ & $3(15,8 \%)$ & $4(44,4 \%)$ & 0,10 \\
\hline Infarto recurrente & $5(17,8 \%)$ & $1(5,3 \%)$ & $4(44,4 \%)$ & 0,01 \\
\hline
\end{tabular}

se encontraron diferencias significativas en la incidencia de eventos cardiovasculares mayores entre ambos grupos. Se constató la muerte de 3 pacientes del grupo epicardico (todos con DP en el estudio índice con IVI) y ninguna en el grupo microvascular $(p<0,01)$. Las muertes consignadas fueron por causa cardiovascular. Ningún paciente presentó un accidente cerebrovascular durante el seguimiento.

\section{Discusión}

Nuestra población de estudio presentó características basales y presentación clínica similares a registros internacionales. Distintas series han comparado poblaciones con IAM con estenosis coronaria aterosclerotica significativa versus MINOCA, señalando en esta última una mayor frecuencia de mujeres, de menor edad al momento de la presentación y prevalencia más baja factores de riesgo cardiovascular típicos ${ }^{5-7}$. En Chile, el grupo de estudios multicéntricos de infarto (GEMI), comparó población con IAM con enfermedad coronaria significativa versus sin enfermedad coronaria significativa, describiendo una mayor proporción de mujeres, menor carga de factores de riesgo cardiovascular y menor presencia de elevación del segmento ST, pero no reportan utilización de IVI ni RMC ${ }^{17}$.

La utilización de IVI es imprescindible para identificar los mecanismos anatómicos intravascu- lares productores de isquemia en estos pacientes. El accidente de placa puede ser categóricamente diagnosticado con IVI, de preferencia $\mathrm{OCT}^{18}$. En nuestro estudio, la mayoría de pacientes fue sometida OCT. Se logró establecer un diagnóstico del mecanismo de MINOCA por IVI en 35,7\% de los pacientes. En un estudio prospectivo con OCT por Opolski y cols. $(\mathrm{n}=38)$, se logró un diagnostico etiológico de MINOCA en $42 \%$ de los pacientes, correspondiendo $24 \%$ a DP y $18 \%$ a trombosis coronaria ${ }^{19}$. En la serie de Reynolds y cols., en que se realizó IVUS y RMC dentro de los primeros 7 días desde el MINOCA $(\mathrm{n}=44), 38 \%$ de los pacientes presentaron $\mathrm{DP}^{20}$. Aunque nuestro trabajo muestra una menor prevalencia de DP, esto probablemente se encuentre condicionado al menor porcentaje de pacientes con trastorno de motilidad con patrón epicardico. Revisiones actuales preconizan la realización de IVI en todos los pacientes con MINOCA y fundamentalmente en el grupo de pacientes con trastorno de motilidad segmentaria con patrón epicárdico ${ }^{18,21}$.

En nuestro estudio, se logró establecer un diagnostico imagenológico en $100 \%$ de los pacientes que contaban con IVI y RMC. Registros internacionales señalan que la RMC permite establecer una caracterización tisular en 74-89\% de los pacientes con MINOCA ${ }^{5,16,22}$. En la serie de Bhatia y cols. $(\mathrm{n}=215)$, la RMC realizada dentro de los primeros 4 días del MINOCA permitió diferenciar el patrón tisular en $83 \%$ pacientes (32\% miocarditis; $22 \%$ infarto; $20 \%$ miocardiopatía no isquémica; y 
9,3\% miocardiopatía por estrés ${ }^{22}$. En la serie de Dastidar y cols. $(\mathrm{n}=388)$, la realización de RMC realizada con una mediana de 37 días desde el evento clínico logro identificar patrón tisular en $74,4 \%$ (25,0\% infarto; $24,7 \%$ miocarditis; $24,7 \%$ miocardiopatía no isquémica) ${ }^{16}$. Los datos de estos estudios se condicen con revisiones sistemáticas de estudios previos a $2015(\mathrm{n}=1.801)$, en que la prevalencia de miocarditis, infarto y miocardiopatías fue de 33\%, 24\% y $23 \%$ respectivamente ${ }^{5}$. La proporción de pacientes de nuestro estudio con miocarditis o infarto constituido por RMC fue menor a los reportes previamente mencionados, probablemente por el menor tamaño de nuestro registro. Las series internacionales han descrito que menos de $3 \%$ de los pacientes con MINOCA se realizan estudio complementario con RMC, por lo que es posible que estas proporciones se modifiquen significativamente al sistematizar el estudio de los pacientes con MINOCA ${ }^{22}$.

Al seguimiento de FEVI, se logró identificar una recuperación significativa en el grupo microvascular versus un aumento no significativo en el grupo epicárdico, lo que representa la evolución clínica de las distintas etiologías, dato no reportado en los registros internacionales. Este dato se relaciona directamente con el pronóstico a largo plazo del paciente ${ }^{23}$.

Al seguimiento clínico reportamos mortalidad solo en el grupo de patrón epicardico y evidencia de DP por IVI, lo que se relaciona directamente a su etiología. Corresponde a una señal de alarma clínica, son un grupo que debe recibir seguimiento y prevención secundaria óptima, ya que la causa es por un accidente de placa, entidad bien caracterizada y conocida en nuestra práctica clínica. Se ha descrito que los pacientes con MINOCA, en comparación a pacientes con enfermedad coronaria aterosclerótica obstructiva, tienen menor probabilidad de recibir terapias médicas de prevención secundaria ${ }^{24,25}$. No reportamos datos respecto de adherencia a medidas de prevención secundaria en los pacientes incluidos en nuestro estudio durante el seguimiento, por no disponer de estos datos clínicos.

\section{Limitaciones}

Al tratarse de un estudio retrospectivo, no se estandariza un flujograma de evaluación de pacientes, lo que explica diferencias en el tipo de IVI utilizada o la ausencia de RMC en algunos pacientes. Se describe una muestra pequeña de pacientes. No se cuenta con datos de test de provocación de vasoespasmo ni estudio de trombofilia de pacientes con estudio negativo por RMC e IVI, no se reporta manejo médico utilizado por los pacientes.

Esto nos motiva a generar un registro prospectivo de estos pacientes con un estudio estandarizado, seguimiento clínico estricto, y optimización de tratamientos médicos y/o necesidad de dispositivos cardiacos en el seguimiento.

\section{Conclusión}

MINOCA es un diagnóstico operativo con múltiples etiologías probables. La población estudiada tuvo predominio de mujeres, con baja carga de factores de riesgo cardiovascular. La utilidad del uso de la multiimagen fue fundamental para el proceso diagnóstico. El balonamiento apical no fue patognomónico de miocardiopatía por estrés. La DP se asoció a mortalidad.

\section{Referencias}

1. Departamento de Estadísticas e Información de Salud. Estadísticas de Salud [Internet]. 2018 [cited 2019 Oct 30]. Available from: https://deis.minsal.cl/estadisticas-de-salud/

2. Roffi M, Patrono C, Collet JP, Mueller C, Valgimigli M, Andreotti F, et al. 2015 ESC Guidelines for the management of acute coronary syndromes in patients presenting without persistent st-segment elevation: Task force for the management of acute coronary syndromes in patients presenting without persistent ST-segment elevation of. Eur Heart J 2016; 37 (3): 267-315.

3. Ibanez B, James S, Agewall S, Antunes MJ, Bucciare1li-Ducci C, Bueno H, et al. 2017 ESC Guidelines for the management of acute myocardial infarction in patients presenting with ST-segment elevation. Eur Heart J 2018; 39 (2): 119-77.

4. Niccoli G, Scalone G, Crea F. Acute myocardial infarction with no obstructive coronary atherosclerosis: mechanisms and management. Eur Heart J 2015; 36 (8): 475-81.

5. Pasupathy S, Air T, Dreyer RP, Tavella R, Beltrame JF. Systematic Review of Patients Presenting With Suspected Myocardial Infarction and Nonobstructive Coronary Arteries. Circulation 2015; 131 (10): 861-70. 
6. Patel MR, Chen AY, Peterson ED, Newby LK, Pollack $\mathrm{CV}$, Brindis RG, et al. Prevalence, predictors, and outcomes of patients with non-ST-segment elevation myocardial infarction and insignificant coronary artery disease: Results from the Can Rapid risk stratification of Unstable angina patients Suppress ADverse outcomes with Early. Am Heart J 2006; 152 (4): 641-7.

7. Planer D, Mehran R, Ohman EM, White HD, Newman JD, Xu K, et al. Prognosis of Patients With Non-ST-Segment-Elevation Myocardial Infarction and Nonobstructive Coronary Artery Disease. Circ Cardiovasc Interv 2014; 7 (3): 285-93.

8. Thygesen K, Joseph Alpert DS, Allan Jaffe US, Chaitman BR, Bax JJ, Morrow DA, et al. 4th Universal Definition of MI. J Am Coll Cardiol 2018; 72 (18): 2231-64.

9. Gościniak P, Baron T, Józwa R, Pyda M. The tip of the iceberg: cardiac magnetic resonance imaging findings in patients with myocardial infarction with non-obstructive coronary arteries: preliminary data from the Polish single-centre registry. Kardiol Pol 2019; 77 (3): 389-92.

10. Smilowitz NR, Mahajan AM, Roe MT, Hellkamp AS, Chiswell K, Gulati M, et al. Mortality of Myocardial Infarction by Sex, Age, and Obstructive Coronary Artery Disease Status in the ACTION Registry-GWTG (Acute Coronary Treatment and Intervention Outcomes Network Registry-Get With the Guidelines). Circ Cardiovasc Qual Outcomes 2017; 10 (12): e003443.

11. Safdar B, Spatz ES, Dreyer RP, Beltrame JF, Lichtman JH, Spertus JA, et al. Presentation, Clinical Profile, and Prognosis of Young Patients With Myocardial Infarction With Nonobstructive Coronary Arteries (MINOCA): Results From the VIRGO Study. J Am Heart Assoc. 2018; 7 (13).

12. Choo EH, Chang K, Lee KY, Lee D, Kim JG, Ahn Y, et al. Prognosis and Predictors of Mortality in Patients Suffering Myocardial Infarction With Non-Obstructive Coronary Arteries. J Am Heart Assoc 2019; 8 (14): e011990.

13. Hicks KA, Mahaffey KW, Mehran R, Nissen SE, Wiviott SD, Dunn B, et al. 2017 Cardiovascular and Stroke Endpoint Definitions for Clinical Trials. Circulation 2018; 137 (9): 961-72.

14. Johnson TW, Räber L, di Mario C, Bourantas C, Jia H, Mattesini A, et al. Clinical use of intracoronary imaging. Part 2: acute coronary syndromes, ambiguous coronary angiography findings, and guiding interventional decision-making: an expert consensus document of the European Association of Percutaneous Cardiovascular Intervent. Eur Heart J 2019; 40 (31): 2566-84.

15. Friedrich MG, Sechtem U, Schulz-Menger J, Holmvang G, Alakija P, Cooper LT, et al. Cardiovascular Magnetic
Resonance in Myocarditis: A JACC White Paper. J Am Coll Cardiol 2009; 53 (17): 1475-87.

16. Dastidar AG, Baritussio A, De Garate E, Drobni Z, Biglino G, Singhal P, et al. Prognostic Role of CMR and Conventional Risk Factors in Myocardial Infarction With Nonobstructed Coronary Arteries. JACC Cardiovasc Imaging 2019; 12 (10): 1973-82.

17. Maldonado R, Madariaga A, López C, Nazzal C, Prieto JC. Caracterización clínica de pacientes con Infarto Agudo al Miocardio sin lesiones coronarias significativas: Estudio GEMI 2011-2013. Rev Chil Cardiol 2014; 33: 165-72.

18. Tamis-Holland JE, Jneid H, Reynolds HR, Agewall S, Brilakis ES, Brown TM, et al. Contemporary Diagnosis and Management of Patients With Myocardial Infarction in the Absence of Obstructive Coronary Artery Disease: A Scientific Statement From the American Heart Association. Circulation 2019; 139 (18): e891-908.

19. Opolski MP, Spiewak M, Marczak M, Debski A, Knaapen P, Schumacher SP, et al. Mechanisms of Myocardial Infarction in Patients With Nonobstructive Coronary Artery Disease. JACC Cardiovasc Imaging 2019; 12 (11): 2210-21.

20. Reynolds HR, Srichai MB, Iqbal SN, Slater JN, Mancini GBJ, Feit F, et al. Mechanisms of myocardial infarction in women without angiographically obstructive coronary artery disease. Circulation 2011; 124 (13): 1414-25.

21. Scalone G, Niccoli G, Crea F. Pathophysiology, diagnosis and management of MINOCA: an update. Eur Hear journal Acute Cardiovasc care 2019; 8 (1): 54-62.

22. Bhatia S, Anstine C, Jaffe AS, Gersh BJ, Chandrasekaran K, Foley TA, et al. Cardiac magnetic resonance in patients with elevated troponin and normal coronary angiography. Heart 2019; 105 (16): 1231-6.

23. Nicod P, Gilpin E, Dittrich H, Chappuis F, Ahnve S, Engler R, et al. Influence on prognosis and morbidity of left ventricular ejection fraction with and without signs of left ventricular failure after acute myocardial infarction. Am J Cardiol 1988; 61 (15): 1165-71.

24. Maddox TM, Ho PM, Roe M, Dai D, Tsai TT, Rumsfeld JS. Utilization of secondary prevention therapies in patients with nonobstructive coronary artery disease identified during cardiac catheterization: Insights from the national cardiovascular data registry cath-PCI registry. Circ Cardiovasc Qual Outcomes 2010; 3 (6): 632-41.

25. Ramanath VS, Armstrong DF, Grzybowski M, Rahnama-Mohagdam S, Tamhane UU, Gordon K, et al. Receipt of cardiac medications upon discharge among men and women with acute coronary syndrome and nonobstructive coronary artery disease. Clin Cardiol 2010; 33 (1): 36-41. 